\title{
Non-invasive measurement of endothelial function: effect on brachial artery dilatation of graded endothelial dependent and independent stimuli
}

Paul Leeson, Sara Thorne, Ann Donald, Michael Mullen, Peter Clarkson, John Deanfield

\begin{abstract}
Objective-To examine the relation between endothelial dependent and endothelial independent stimuli of varying intensity and measures of vascular function in the brachial artery of young healthy adults, to determine whether these responses are consistent and can be used to assess endothelial function.

Design and setting-High resolution ultrasound was used to measure brachial artery diameter at rest, after reactive hyperaemia induced by forearm cuff occlusion, and after sublingual isosorbide dinitrate (ISDN). Each subject was assigned to a random order of six cuff occlusion times (30 seconds, $1 \cdot 5,2 \cdot 5,3 \cdot 5$, $4 \cdot 5$, and 8 minutes) and six doses of ISDN $(10,20,50,100,200$, and $400 \mu \mathrm{g})$.
\end{abstract}

Subjects-Three males and three females mean age 31 years (range 25 to 43 ) with no known risk factors for cardiovascular disease.

Main outcome measures-Flow mediated, endothelial dependent dilatation was compared to duration of cuff occlusion, peak reactive hyperaemia, and duration of increased flow. Nitrate induced, endothelial independent dilatation was compared to dose of ISDN and change in flow.

Results-Vessel dilatation and duration of peak flow change increased with longer duration of cuff occlusion. After $4.5 \mathrm{~min}$ utes of occlusion, flow mediated dilatation was mean (SD) 96 (6)\% of maximal response and did not increase significantly with longer occlusion times. No significant dilatation occurred after 10 or $20 \mu \mathrm{g}$ of ISDN in any subject. With increasing doses up to $200 \mu \mathrm{g}$ there was an increase in dilatation. Endothelial independent dilatation did not increase significantly with doses of ISDN above $200 \mu \mathrm{g}$.

Conclusions-Measures of arterial function vary with duration of blood flow occlusion and ISDN dose. Maximum arterial response was reached in all subjects after 4.5 minutes of blood flow occlusion or $200 \mu \mathrm{g}$ of ISDN. Therefore, these variables produce consistent reproducible measures of endothelial function.
(Heart 1997;78:22-27)

Keywords: endothelium; vascular reactivity; atherogenesis

Endothelial dysfunction plays a key part both in the pathophysiology of established coronary atherosclerosis and in the early stages of atherogenesis, before the onset of clinical disease. ${ }^{1-3}$ There is, therefore, increasing interest in the study of endothelial function in young subjects with cardiovascular risk factors ${ }^{4}$, to develop effective interventions at an early stage with the aim of altering the natural history of vascular disease. ${ }^{5}$ Strain gauge venous occlusion plethysmography has been used to study small vessel reactivity in response to pharmacological or physiological stimuli ${ }^{7}$ but is usually invasive and not able to examine large vessel physiology which is more relevant to vascular disease. We have developed a noninvasive high resolution ultrasound protocol to characterise endothelial dependent responses in the conduit arteries of the arm and leg. ${ }^{4910}$ This compares dilatation to increased blood flow (an endothelial dependent response) ${ }^{3}$ following reactive hyperaemia induced by cuff inflation and deflation on the distal limb, with response to a sublingual nitrate, which is independent of endothelial function.

Impaired flow mediated dilatation has been shown in subjects as young as 7 years old with familial hypercholesterolaemia, ${ }^{9}$ in patients with insulin dependent diabetes, and in active $^{10}$ and passive cigarette smokers, ${ }^{11}$ suggesting early endothelial dysfunction. However, the relation between the intensity of the dilating stimulus (flow or nitrate) and the degree of vascular reactivity, which may influence interpretation of the biological significance of these experiments, has not been studied.

We examined the relation between dilatation of the brachial artery and differing duration of cuff occlusion (to influence the degree of flow increase) and isosorbide dinitrate (ISDN) doses, to establish the optimum duration of blood flow occlusion and nitrate dose to obtain maximum response to the two stimuli. This information will facilitate the use of this non-invasive measure of vascular function 
in serial studies of interventions designed to reverse early arterial damage.

\section{Methods}

SUBJECTS AND VISITS

Six adult volunteers (three males and three females) with a mean age of 31 years (range 25 to 43) were recruited from our institution. All were normotensive, non-smokers, with normal serum cholesterol, no history of diabetes or family history of premature vascular disease, and none was receiving vasoactive medications.

Each subject underwent six separate vascular studies to assess brachial artery response to flow increase and ISDN. On each occasion on separate days they received one of six cuff occlusion times ( 30 seconds, $1.5,2.5,3.5$, $4 \cdot 5$, and 8 minutes) and one of six sublingual ISDN doses (Isoket, Schwarz Pharma Ltd, Buckinghamshire, UK) $(10 \mu \mathrm{g}, 20 \mu \mathrm{g}, 50 \mu \mathrm{g}$, $100 \mu \mathrm{g}, 200 \mu \mathrm{g}$, and $400 \mu \mathrm{g})$. These studies were performed in a randomised order for each subject.

\section{ULTRASOUND SCAN TECHNIQUE}

Ultrasound scans were performed with subjects supine, at rest and the recorded room temperatures ranged from $20^{\circ} \mathrm{C}$ to $26^{\circ} \mathrm{C}$. Blood pressure was measured using a standard sphygmomanometer. On each visit, a B mode scan of the right brachial artery was obtained in longitudinal section between $5 \mathrm{~cm}$ and $10 \mathrm{~cm}$ above the elbow using a $7.0 \mathrm{MHz}$ linear array transducer and a standard Acuson $128 \mathrm{XP} / 10$ system. The centre of the artery was identified when the clearest picture of the anterior and posterior intimal layers was obtained. The transducer was held at the same point on the upper arm throughout the scan, using a stereotactic clamp. The position of the transducer relative to the antecubital fossa was recorded on the first visit and the same point used at each subsequent scan. The transmit (focus) zone was set to the depth of the anterior wall of the right brachial artery. Depth and gain settings were set to optimise images of the lumen-arterial wall interface, images were magnified using a resolution box function, and operating parameters were not changed during the study. To measure arterial diameter the clearest defined section of the artery on B mode imaging was identified and an $M$ mode cursor positioned over this point, at right angles to the arterial lumen. A five second segment of the A mode signal was then routed to a Wall Tracking System (Ingenious Systems, The Netherlands) which automatically tracks vessel wall movement on a beat by beat basis. The minimum arterial diameter was calculated from the distance between opposite lumen-arterial wall interfaces, as identified by manual selection of the maximal change in recorded radio frequency amplitude. ${ }^{12-14}$ Repeated measures in an individual using this system have been shown to be consistent and reproducible. ${ }^{14} 15$

A resting scan was recorded, and arterial flow velocity measured using a pulsed
Doppler signal at $70^{\circ}$ to the vessel with the range gate $(1.5 \mathrm{~mm})$ in the centre of the artery. A pneumatic cuff, placed around the forearm distal to the imaged brachial artery segment, was then inflated to a pressure of $300 \mathrm{~mm} \mathrm{Hg}$ for the length of time indicated by the study protocol. A second scan was taken continuously from 30 seconds before to 70 seconds after cuff release. This enabled flow velocity to be recorded for 10 seconds before and for 30 seconds after release, and a second measure of vessel diameter to be made between 55 and 65 seconds after cuff deflation. Ten minutes was allowed for vessel recovery, after which a second resting control scan and flow measurement was taken. ISDN was then administered according to the protocol and three minutes later a fourth scan of the vessel, with a final flow measurement, was recorded.

\section{DATA ANALYSIS}

The scans were assessed blindly from video tape playback and hard copy records of arterial diameter measurements. If the $M$ mode cursor was not at the same point on the artery throughout the scan or if mean diameter measurements on the hard copy record had a variability of $>0.06 \mathrm{~mm}$ the whole study was discarded and repeated. The main outcome measure from the scans was flow mediated dilatation which is expressed as the percentage change in vessel diameter post hyperaemia. Similarly, response to ISDN was defined as the percentage change in diameter after ISDN.

Volume flow was calculated by multiplying the velocity time integral of the Doppler flow signal (corrected for angle) for a single pulse wave by the heart rate and vessel crosssectional area. The peak response to reactive hyperaemia was recorded as the maximum flow in a single cardiac cycle within the first 15 seconds after cuff deflation, divided by the flow during the resting (baseline) scan. Volume flow was also measured every five seconds for the 30 seconds immediately following forearm cuff deflation.

To examine dose response effects of length of cuff occlusion, the degree of flow mediated dilatation at eight minutes (the longest cuff occlusion time studied) was assumed to be maximal and the other flow mediated dilatations calculated as a percentage of the eight minute value. Similarly, dilatation after $400 \mu \mathrm{g}$ of ISDN was assumed to be maximal and values for other doses were expressed as a percentage of the response to $400 \mu \mathrm{g}$. To minimise the influence of age and sex equal numbers of male and female subjects of similar ages were studied (table). Room temperature, time of day, body mass index, and vessel size were also similar within the group and did not appear to alter the vascular responses to flow and ISDN. We investigated the influence of cuff occlusion time and dose of ISDN on vessel diameter change and peak flow increase, and of cuff occlusion time on the volume of increased flow in the 15 seconds after cuff release. 
Characteristics of study group (mean (1 SD))

\begin{tabular}{lc}
\hline Number & 6 \\
Age (years) & 31 (range 25 to 43) \\
Cholesterol (mmol/l) & $4 \cdot 6(0 \cdot 7)$ \\
Systolic blood pressure (mm Hg) & $123(14)$ \\
Diastolic blood pressure (mm Hg) & $71(11)$ \\
Height (cm) & $173(12)$ \\
Weight (kg) & $64(13)$ \\
\hline
\end{tabular}

\section{Results}

Thirty eight studies were performed (six per subject and two repeated for technical reasons) and were well tolerated. On two occasions, subjects complained of a slight headache following $400 \mu \mathrm{g}$ ISDN. None of the subjects complained of major discomfort following forearm cuff occlusion apart from transient change in sensation following cuff release, which was more frequently experienced with longer cuff occlusion times.

\section{DURATION OF CUFF OCCLUSION AND FLOW} MEDIATED DILATATION

Flow mediated dilatation was dependent on the duration of cuff occlusion (figs 1 and 2).

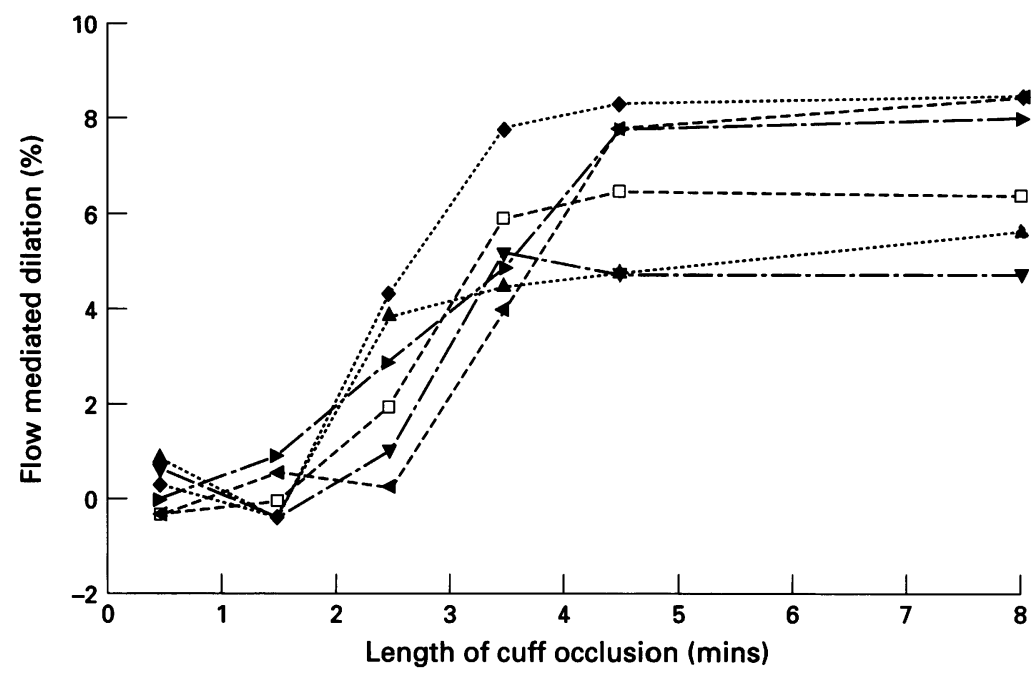

Figure 1 Relation between duration of forearm cuff occlusion and percentage flow mediated dilatation. Each line represents the response of a single subject.

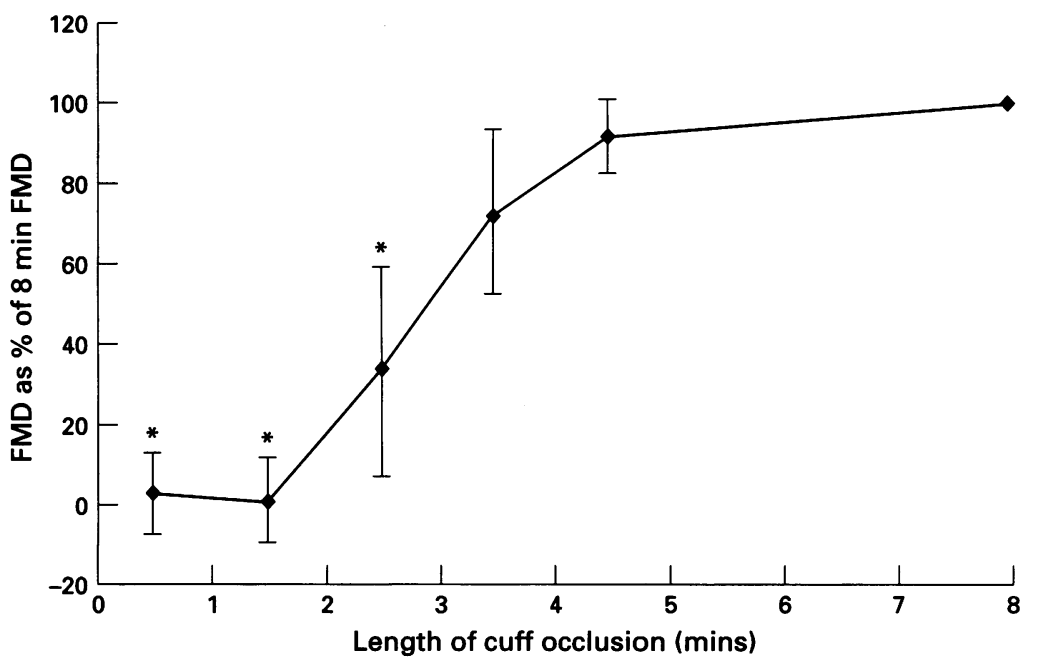

Figure 2 Relation between duration of forearm cuff occlusion and flow mediated dilatation (FMD). FMD at each cuff occlusion time is shown as a percentage of the maximal FMD after eight minutes. Error bars are $1 S D$. ${ }^{\star}$ Duration of cuff occlusions where the $F M D$ is significantly different $(P<0.05)$ from the $F M D$ after eight minutes.
No significant dilatation was seen for cuff occlusion times of less than 1.5 minutes. With increasing cuff occlusion times, up to $4.5 \mathrm{~min}$ utes, flow mediated dilatation increased linearly. For 2.5 minutes of occlusion, flow mediated dilatation was mean (SD) 36 (23)\% of maximum and at 3.5 minutes it was 81 (22)\%. Three subjects had reached $>90 \%$ of their maximum flow mediated dilatation following 3.5 minutes occlusion, and after 4.5 minutes of occlusion the whole group had reached 96 (6)\% of maximum flow mediated dilatation. This pattern was highly consistent and no significant increase was seen in any subject after cuff occlusion times greater than 4.5 minutes. Mean difference between flow mediated dilatation at 4.5 minutes and at 8 minutes was $-0.3 \%$, (95\% confidence interval (CI) -0.07 to $0 \cdot 10 \%)$.

\section{DURATION OF CUFF OCCLUSION AND PEAK} REACTIVE HYPERAEMIA

Peak reactive hyperaemia (within 15 seconds of cuff release) increased with cuff occlusion time up to 1.5 minutes, rising from $60(28) \%$ of maximal reactive hyperaemia after 30 seconds to 98 (26)\% after 1.5 minutes. Cuff occlusion times longer than 1.5 minutes did not significantly increase the peak reactive hyperaemia measurements (reactive hyperaemia after 4.5 minutes of cuff occlusion was 105 (10)\% of the eight minute cuff occlusion reactive hyperaemia). This differs from the relation between cuff occlusion time and flow mediated dilatation which continued to increase with cuff occlusions of up to 4.5 minutes.

\section{DURATION OF CUFF OCCLUSION AND}

\section{DURATION OF REACTIVE HYPERAEMIA}

Cuff occlusions of 1.5 minutes or longer resulted in similar peak reactive hyperaemias of between $200 \%$ and $280 \%$ of baseline flow levels during the first five seconds after cuff release (fig 3). The duration of this peak change, however, differed depending on the length of cuff occlusion: 3.5 minutes or longer produced a prolonged peak reactive hyperaemia resulting in significantly higher flow levels 15 seconds after cuff release compared with 1.5 or 2.5 minutes of cuff occlusion (mean difference between flow increase at 8 minutes and 2.5 minutes was $91 \%(95 \%$ CI $1.6-$ $180 \%)$ ).

As a means of characterising the intensity and duration of flow increase over the first 15 seconds after cuff release we quantified the integral of flow (area under the curve) of this flow change, a measure used previously in flow studies. ${ }^{16}$ There was a relation between the integral of flow over the first 15 seconds after cuff release and the degree of sustained vessel dilatation measured after one minute (fig 4). At least 4.5 minutes of cuff occlusion was required to cause maximal increase in flow over the 15 seconds, corresponding with our finding that 4.5 minutes of cuff occlusion was required to cause maximal vessel dilatation one minute after cuff release. The analysis of flow increase and integral of flow was repeated at 20 and 30 seconds after cuff release with 


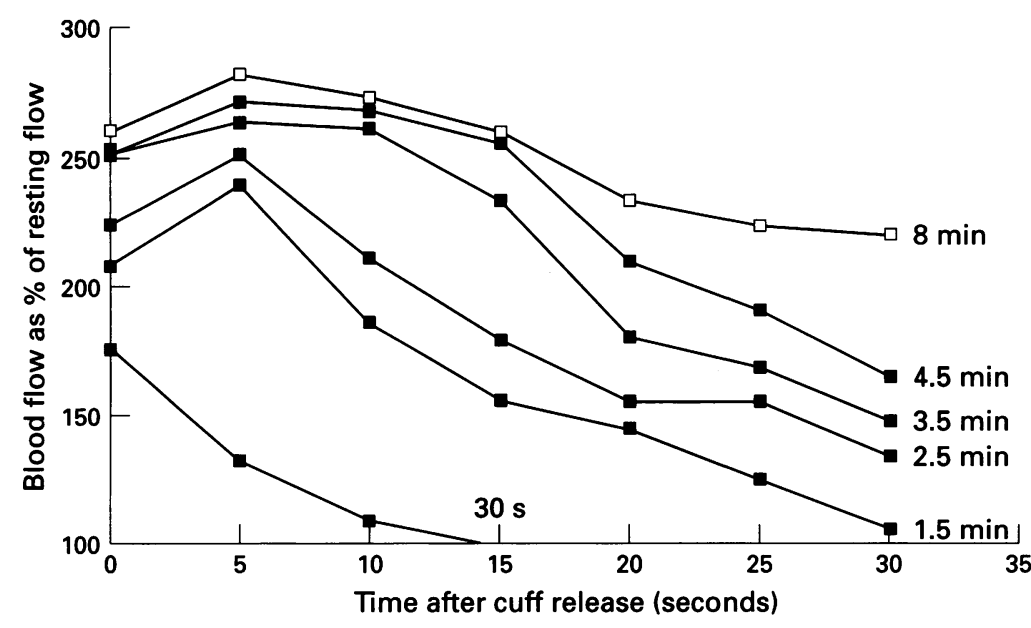

Figure 3 Change in flow increase for the 30 seconds after cuff release at each duration of cuff occlusion. All durations greater than 30 seconds induce similar peak response after around five seconds; however, at least 3.5 minutes of cuff occlusion are required for a sustained increase after 15 seconds.

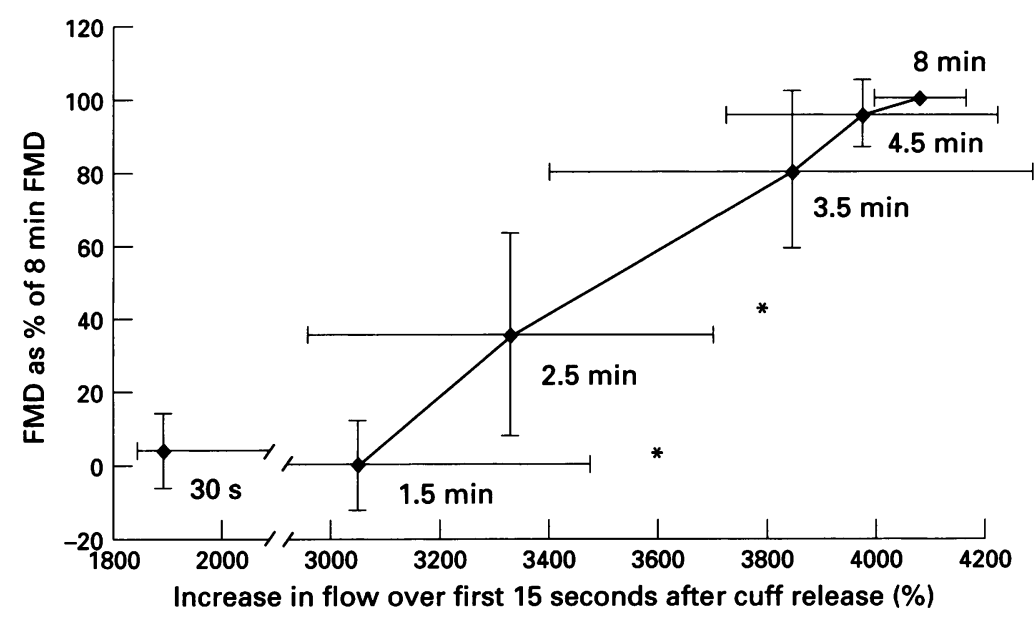

Figure 4 Relation between integral of flow increase for the 15 seconds after cuff release and the degree of vessel dilatation at each duration of cuff occlusion. Maximal vessel dilatation at one minute after cuff release does not occur until maximal flow increase has been induced after at least 3.5 minutes of cuff occlusion. ${ }^{\star}$ Degrees of flow increase significantly different $(P<0.05)$ from the flow increase after eight minutes.

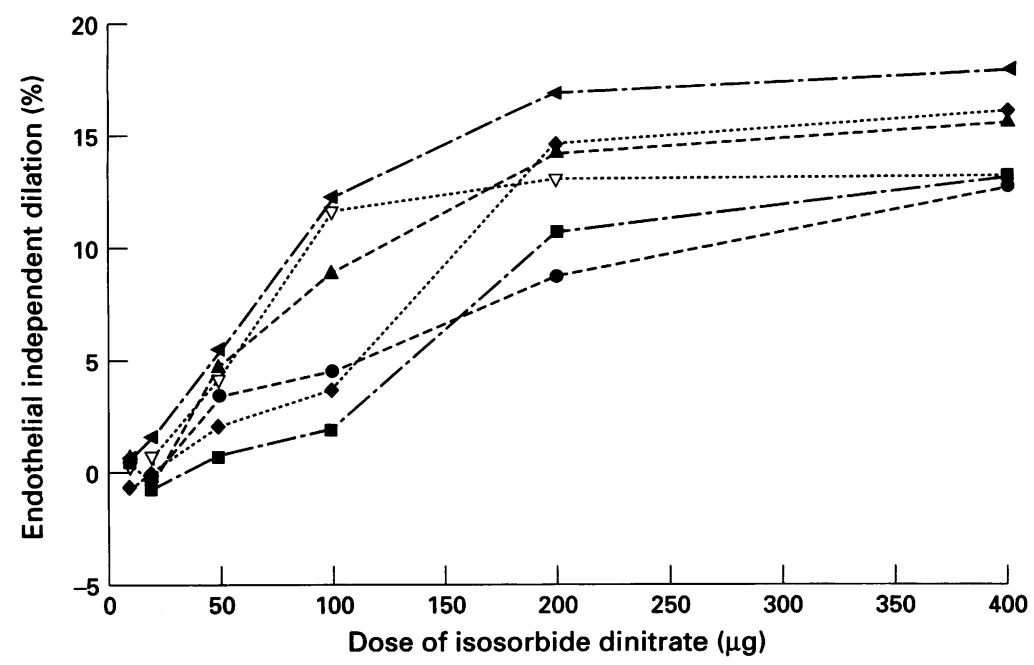

Figure 5 Relation between isosorbide dinitrate dose and percentage endothelial independent dilatation. Each line represents the response of a single subject. similar findings. The main difference was at 30 seconds when increase in flow had returned to baseline if 1.5 minutes of cuff occlusion had been used.

DOSE OF ISDN AND DILATATION

The degree of dilatation following ISDN was dependent on the dose (figs 5 and 6 ). No significant dilatation occurred after $10 \mu \mathrm{g}$ (3.5 $(3.5) \%$ of dilatation following $400 \mu \mathrm{g})$ or $20 \mu \mathrm{g}$ (1 (6)\% of dilatation following $400 \mu \mathrm{g}$ ) in any subject. With increasing doses up to $200 \mu \mathrm{g}$ there was an increase in dilatation. Response after $200 \mu \mathrm{g}$ of ISDN was 89 (13)\% of the maximum arterial dilatation after $400 \mu \mathrm{g}$. No further increase in diameter change was seen at higher doses. Mean difference in dilatation between $200 \mu \mathrm{g}$ and $400 \mu \mathrm{g}$ of ISDN was $-1.5 \%(95 \%$ CI -3.28 to $0 \cdot 26 \%$ ). Brachial artery flow increase was linearly related to ISDN dose.

\section{Discussion}

The ability to study changes in vascular endothelial function has important and widespread clinical applications for the development of strategies aimed at modifying the progression of atherosclerosis. Endothelial dysfunction has been inferred in invasive protocols when responses to pharmacological agents or physiological stimuli acting via the endothelium, such as acetylcholine or flow, have been impaired relative to the vessel's response to a nitrate, ${ }^{23}$ which acts directly on vascular smooth muscle. We used this experimental principle to develop a non-invasive approach which has been used in cross-sectional studies of young preclinical subjects ${ }^{415}$ and interventional investigations designed to restore endothelial function ${ }^{56}$ at a much earlier stage in disease development. Interpretation of such studies depends on differences between endothelial and non-endothelial responses being caused by altered vascular biology rather than merely a reflection of the intensity of stimulus. In this study we have shown that maximum degrees of endothelial dependent and independent dilatation are achieved in normal subjects when 4.5 minutes of cuff occlusion are compared to $200 \mu \mathrm{g}$ of ISDN. Non-invasive investigations using these parameters, therefore, provide an objective, experimental assessment of endothelial response which can be used to characterise vascular physiology in a wide variety of clinical situations.

Flow mediated dilatation is a localised vascular response which has been demonstrated in animals ${ }^{17}$ and humans and occurs in all large arteries. ${ }^{1819}$ It is dependent on the endothelium as arteries stripped of the endothelial lining lose their ability to dilate following increased flow. ${ }^{20}$ Furthermore, it appears this response is partly mediated via nitric oxide produced from the endothelium as vasodilatation following increased flow can be inhibited by an intra-arterial infusion of the competitive inhibitor of nitric oxide production L-NMMA. ${ }^{21} 22$ This flow related nitric 


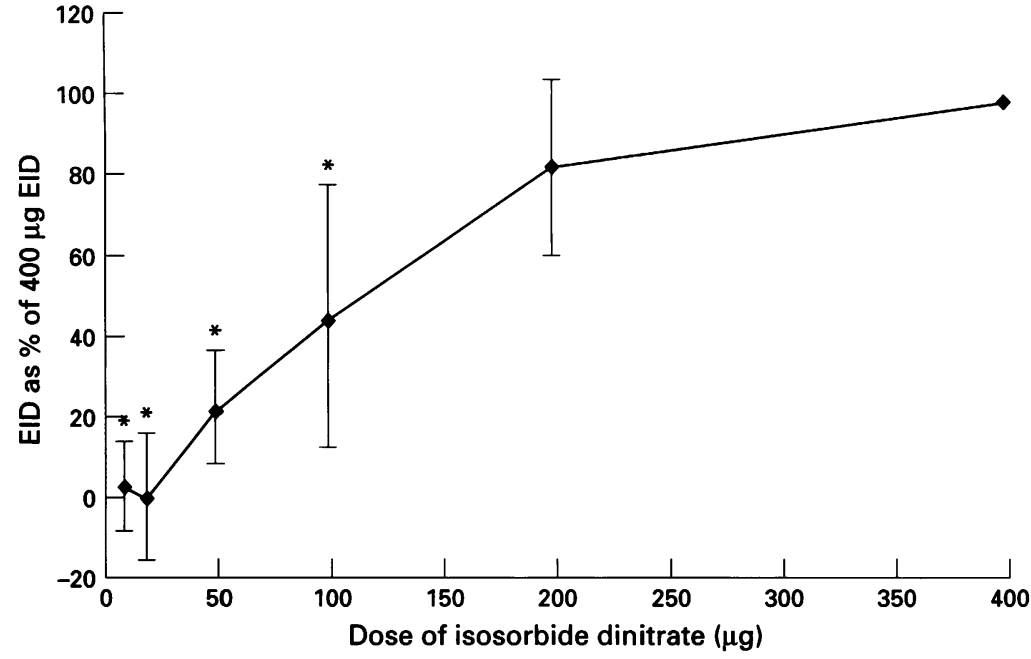

Figure 6 Relation between dose of isosorbide dinitrate and endothelial independent dilatation (EID). EID at each dose is shown as a percentage of the maximal EID after $400 \mu \mathrm{g}$. Error bars represent $1 S D$. ${ }^{\star}$ Dose of isosorbide dinitrate where the EID is significantly different $(P<0.05)$ from the EID after $400 \mu \mathrm{g}$.

oxide release is thought to be triggered by increased shear stress on the vessel wall after flow increase. ${ }^{23}$ Previously, when measuring vascular reactivity, it was assumed that a maximal flow stimulus had been provided if there was a peak change in flow over one cardiac cycle following cuff release. ${ }^{4}$ However, the present study shows that peak flow change reaches a maximum after 1.5 minutes of cuff occlusion whereas maximum vessel dilatation does not occur until after 4.5 minutes of cuff occlusion. The length of cuff occlusion required to produce peak flow has been demonstrated in previous studies using both venous occlusion plethysmography ${ }^{19}$ and Doppler based systems ${ }^{24}$ which also show peak flow changes after as little as one minute of cuff occlusion. Measures of peak reactive hyperaemia, however, do not provide any information about the duration of flow increase. Short periods of cuff occlusion may produce maximal reactive hyperaemia but the duration of this peak flow increase may be attenuated compared with that of longer cuff occlusion times. Therefore, shorter durations of cuff occlusion provide a less intense stimulus which fails to reach the threshold to cause sustained vasodilatation identifiable one minute after cuff release. To assess this possibility we analysed our experiments to examine the duration of peak flow and the integral of the flow increase (the change in volume of flow through the artery during the 15 seconds after cuff release). We found that although $1.5 \mathrm{~min}$ utes and 2.5 minutes of cuff occlusion induced a peak response, this increase in flow began to fall after five seconds, whereas after cuff occlusion times of 3.5 minutes or longer, flow remained at peak levels until at least $15 \mathrm{sec}-$ onds after cuff release. If the integral of flow over 15 seconds (area under the curve) is plotted against vessel dilatation there is a relation showing that above a threshold stimulus after 1.5 minutes of cuff occlusion there is an association between flow and vessel dilatation. Maximal vessel dilatation and maximal flow change only occurs after at least 4.5 minutes of cuff occlusion. The practical implication of these findings are that it is important to ensure a cuff time of 4.5 minutes to provoke a sustained flow increase and a reproducible maximum flow mediated dilatation and that higher cuff occlusion times do not provide greater response. Also, that peak reactive hyperaemia alone does not adequately define the intensity of stimulus causing vasodilatation.

Nitrate containing drugs act as an exogenous source of nitric oxide to cause arterial smooth muscle relaxation directly, independent of the endothelium. ${ }^{25}$ This study shows that response to sublingual ISDN is maximum after $200 \mu \mathrm{g}$ and that higher doses do not produce greater dilatation. ISDN was used in this study instead of glyceryl trinitrate (our standard nitrate donating drug) because it was easier to dilute for the dose response study and more stable in lower concentrations. ${ }^{26}{ }^{27}$ All ISDN concentrations used provide smaller nitrate doses than the single sublingual spray of glyceryl trinitrate we have reported previously ${ }^{28}$; however, the response to this stimulus in the same subjects is comparable to the maximal effect seen after $200 \mu \mathrm{g}$ of ISDN. The submaximal $100 \mu \mathrm{g}$ dose of ISDN produces the equivalent degree of vasodilatation as the maximal endothelial dependent response. These results highlight the fact that the brachial artery has the potential to dilate more when stimulated by pharmacological doses of nitrate acting independently of the endothelium than following a physiological stimulus.

Loss of normal endothelial function may occur in young subjects and be an important pathogenic mechanism for the development of atherosclerosis. Endothelial derived nitric oxide is important in regulation of blood flow and inhibits platelet aggregation and monocyte adhesion. We used the non-invasive technique described in this study to demonstrate impaired flow mediated dilatation and relative preservation of response to glyceryl trinitrate, in preclinical subjects with a wide range of risk factors known to predispose to arterial disease in later life. The results now reported further support the interpretation of these findings that loss of flow mediated dilatation is due to a specific pathophysiological change in endothelial function. This non-invasive protocol, which is accurate and reproducible, ${ }^{28}$ has been shown to produce consistent physiological and pharmacological arterial responses and is ideal for evaluation of interventions designed to alter favourably the progression of arterial disease in young people.

CPM Leeson is supported by a Medical Research Counci Studentship, AE Donald by a grant from CORDA, and $M$ Mullen by a Bristol-Myers Squibb Cardiovascular Research Fellowship.

1 Ross R. The pathogenesis of atherosclerosis-perspective for the 1990s. Nature 1993;362:801-9.

2 Ludmer PL, Selwyn AP, Shook TL. Paradoxical vasoconstriction induced by acetylcholine in atherosclerotic coronary arteries. $N$ Engl $\mathcal{F}$ Med 1986;315:1046-51.

3 Nabel EL, Selwyn AP, Ganz P. Large coronary arteries in humans are responsive to changing blood flow: an endothelium-dependent mechanism that fails in patients with atherosclerosis. F Am Coll Cardiol 1990;16:349-56. 4 Celermajer DS, Sorenson KE, Gooch VM, Spiegelhalte 
DJ, Miller OI, Sullivan I, et al. Non-invasive detection of endothelial dysfunction in children and adults at risk of atherosclerosis. Lancet 1992;340:1111-15.

5 Clarkson P, Montgomery H, Donald A, Powe A, Bull T, Dollery C, et al. Exercise training enhances endothelial function in young men [abstract]. $\mathcal{F} \mathrm{Am}$ Coll Cardiol 1996;27(2):288A

6 Clarkson P, Adams MR, Powe AJ, Donald AE, McCredie $\mathrm{R}$, Robinson J, et al. Oral L-arginine improves endothelium-dependent dilation in hypercholesterolaemic young adults. Circulation 1996;97:1989-94.

7 Calver A, Collier J, Vallance P. Inhibition and stimulation of nitric oxide synthesis in the human forearm arterial bed of patients with insulin dependent diabetes. $\mathcal{f}$ Clin Invest 1992;90:2548-54.

8 Whitney RJ. The measurement of volume changes in human limbs. F Physiol 1953;121:1-27.

9 Sorenson KE, Celermajer DS, Georgakopoulos D, Hatcher G, Betteridge J, Deanfield JE. Impairment of endothelium-dependent dilation is an early event in children with familial hypercholesterolaemia and is related to lipoprotein (a) level. $\mathcal{f}$ Clin Invest 1994;93:50-5.

10 Celermajer DS, Sorenson KE, Georgakopoulos D, Bull C, Thomas O, Robinson J, et al. Cigarette smoking is associated with dose related and potentially reversible impairment of endothelium-dependent dilation in healthy young adults. Circulation 1993;88:2149-55.

11 Celermajer DS, Adams MR, Clarkson P, Robinson J, McCredie R, Donald A, et al. Passive smoking and impaired endothelium-dependent arterial dilatation in healthy young adults. $N$ Engl $f$ Med 1996;334:150-4.

12 Kool MJF, Hoeks APG, Struijker Boudier HAJ, Reneman RS, VanBortel LMAB. Short- and long-term effects of smoking on arterial wall properties

13 Hoeks APG, Brands PJ, Smeets FAM, Reneman RS. Assessment of the distensibility of superficial arteries. Assessment of the distensibility of
Ultrasound Med Biol 1990;16:121-8.

14 Ramsey MW, Goodfellow J, Jones CJH, Luddington LA, Lewis MJ, Henderson AH. Endothelial control of arterial distensibility is impaired in chronic heart failure. Circulation 1995;92:3212-19.

15 Leeson $P$, Whincup P, Cook D, Totenhofer R, Donald AE, Lucas A, et al. Birthweight is related to endothelial func- tion in systemic arteries of children [abstract]. $7 \mathrm{Am}$ Coll Cardiol 1996;27(2):275A.

16 Hornig B, Maier V, Drexler H. Physical training improves endothelial function in patients with chronic heart failure. Circulation 1996;93:210-14.

17 Hintze TH, Vatner SF. Reactive dilatation of large coronary arteries in conscious dogs. Circ Res 1984;54:50-7.

18 Anderson EA, Mark SL. Flow mediated and reflex changes in large peripheral artery tone in humans. Circulation 1989;79:93-100.

19 Sinoway LI, Hendrickson C, Davidson WR, Propler S, Zelis $R$. Characteristics of flow mediated brachial artery vasodilatation in human subjects. Circ Res 1989;64:32-42.

20 Pohl U, Holtz J, Busse R, Baseenge E. Crucial role of endothelium in the vasodilator response to increased flow in vivo. Hypertension 1986;8:37-44.

21 Chu A, Chambers DE, Lin CC, Kuehi WD, Palmer RJ, Moncada S, Cobb FR. Effects of inhibition of nitric oxide formation on basal vasomotion and endotheliumdependent responses of coronary arteries in awake dogs. $\mathcal{F}$ Clin Invest 1991;87:1964-8.

22 Joannides $\mathrm{R}$ Haefeli WE, Linder $\mathrm{L}$ Richard $\mathrm{V}$, Bakkali EH, Thuillez C, Luscher TF. Nitric oxide is responsible for flow-dependent dilatation of human periphera

23 Busse R, Fleming I, Heckler M. Signal transduction in endothelium-dependent vasodilatation. Eur Heart $\mathcal{f}$ 1993;14:12-19.

24 Levenson J, Simon A, Pithois-Merli I. Brachial artery changes in response to wrist occlusion in normotensive and hypertensive men. Am $\mathcal{F}$ Physiol 1987;253:217-24.

25 Lerman A, Burnett JC. Intact and altered endothelium in regulation of vasomotion. Circulation 1992;86(suppl III): III12-19.

26 Anderson KA. A practical guide to nitrates. Postgrad Med 1991;89:67-77.

27 Abrams J. Glyceryl trinitrate (nitroglycerin) and the organic nitrates: choosing the method of administration. Drugs 1987;34:391-483.

28 Sorenson KE, Celermajer DS, Spiegelhalter DJ, Georgakopoulos D, Robinson J, Thomas O, et al. Non invasive measure of human endothelium-dependent arteinvasive measure of human endothelium-dependent arte-
rial responses: accuracy and reproducibility. $\mathrm{Br} \mathrm{Heart} \mathcal{f}$ 1995;74:247-53. 